\title{
Oxidatively Modified Carbon as Efficient Material for Removing Radionuclides from Water
}

Artur Khannanov, ${ }^{+}$Vadim V. Nekljudov, ${ }^{+}$Bulat Gareev, ${ }^{+}$Airat Kiiamov, ${ }^{+}$James M. Tour, ${ }{ }$ and Ayrat M. Dimiev ${ }^{+*}$

${ }^{+}$Laboratory of Advanced Carbon Nanomaterials, Kazan Federal University, Kremlyovskaya Street 18, Kazan 420008, Russian Federation;

"Department of Chemistry, Department of Material Science and NanoEngineering, and NanoCarbon Center, Rice University, MS-222, 6100 main Street, Houston TX, 77005, USA; Corresponding authors: e-mail AMDimiev@kpfu.ru (Ayrat M. Dimiev)and tour@ rice.edu (James M. Tour)

There is a constant need to develop advantageous materials for removing radioactive waste from aqueous systems. Here we propose a new carbon-based material prepared by oxidative treatment of various natural carbon sources. The as-prepared oxidatively modified carbon $(O M C)$ has an oxygen-rich surface, and retains its particulate granular texture. It has relatively low cost and can be used in traditional filtration columns. The sorption ability of OMC toward several metal cations is demonstrated. It is especially efficient toward $\mathrm{Cs}^{+}$cations, the species that are among the most difficult to remove from the waters at the Fukushima nuclear plant.

\section{Introduction}

Radioactive waste imposes a serious problem to modern society, threatening life and health of many worldwide. The recent accident at the Fukushima nuclear plant in Japan demonstrated the magnitude of the problem. ${ }^{1}$ Currently, millions of tons of water contaminated by radionuclides are stored in massive tanks on the plant site awaiting efficient methods of purification. Among the radionuclides most difficult to remove from water is ${ }^{137} \mathrm{Cs}^{+} .1$ The ease with which radionuclides can be removed from water decreases in the order of trivalent, divalent to monovalent metal cations. While trivalent elements such as $\mathrm{U}^{3+}$ can be almost quantitatively removed by numerous known absorbents, the sorption of monovalent ${ }^{137} \mathrm{Cs}^{+}$remains an enormous challenge. ${ }^{1-4}$ Sorption with porous minerals, ${ }^{5-10}$ and ion-exchange media ${ }^{11}$ are among the most effective methods for the $\mathrm{Cs}^{+}$removal.

The only known materials to efficiently sorb $\mathrm{Cs}^{+}$are synthetic zeolites. ${ }^{5-10}$ However, the relatively high cost of synthetic zeolites lowers their performance-to-cost ratio. In addition, after 
sorption, $\mathrm{Cs}^{1+}$ cannot be removed from zeolites as easy as from the ion-exchange resins. Thus, the spent zeolites (or similar mineral absorbents) need to be compartmentalized and stored along with sorbed radionuclides until their near complete decomposition by the natural radioactive decay.

From this perspectives, carbon-based materials have advantage over the clays and minerals. In practice, after sorption, carbon can be burned in a nuclear furnace leaving only radioactive ash, which results in an ultra-small volume of radioactive waste. Alternatively, carbon-based materials can be regenerated and be used in a new purification cycle. But traditional carbonbased absorbents such as activated carbon and charcoal are not efficient scavengers of metal cations. While being effective in gaseous phases and for removing some organic contaminants from water, they do not efficiently bind metal cations from aqueous solutions. For this purpose, the carbon surface needs to be decorated with oxygen functional groups.

Recently, the method of removing radionuclides by graphene oxide (GO) was reported. ${ }^{12} \mathrm{GO}$ was demonstrated to be very efficient toward several trivalent and divalent metal cations due to the presence of oxygen functional groups, and due to its high surface area. However, the sorption of monovalent ${ }^{137} \mathrm{Cs}^{+}$was not demonstrated in the above-mentioned study. ${ }^{12}$ In general, the affinity of GO toward monovalent metal cations is lower. ${ }^{13}$ In addition, the usage of GO as a sorbent suffers from the difficulties with the practical implementation: in water, GO forms a stable colloidal solution, which is difficult to separate by common methods. Another problem, preventing application of GO in general, is its high cost. Thus, new materials are needed for purifying water from metal cations. This new material should be as efficient in sorption as GO, but significantly cheaper than it. Also it should be easily separable from water to be costeffective in practical implementation. Ideally, it should have granular particulate texture to be able to be used as a stationary phase in traditional sorption columns.

Here we propose a new material which we term oxidatively modified carbon (OMC) that possesses both low cost and an ability to be easily separated from water. OMC can be prepared from various forms of carbon by treatment with strong oxidants in concentrated acid media in conditions similar to those for the preparation of GO. ${ }^{13-17}$ This treatment generates numerous oxygen functional groups on the surface of the existing pores, making the surface chemically similar to that of GO. Unlike GO, OMC is three-dimensional, and it does not exfoliate. While 
GO production from graphite requires three to six weight equivalents (wt equiv) of $\mathrm{KMnO}_{4}$ per one part of graphite, ${ }^{14,17}$ production of OMC requires only 0.9 to 1.2 wt equiv of $\mathrm{KMnO}_{4}$. Moreover, oxidative treatment can also be effected by a $\mathrm{HNO}_{3} / \mathrm{H}_{2} \mathrm{SO}_{4}$ mixture, completely avoiding the use of $\mathrm{KMnO}_{4}$. After oxidation, carbon retains its particulate nature; the reaction mixture is not thickened. Subsequently, the reaction requires $7 \mathrm{x}$ less $\mathrm{H}_{2} \mathrm{SO}_{4}$ compared to that needed for GO production. Finally and most importantly, during the purification steps, separation of as-prepared OMC from the acidic waste waters can be conducted by simple filtration. This makes the cost of OMC $\sim 10 \mathrm{x}$ lower than that of GO.

Various natural carbon sources can be used for OMC preparation. Depending on the source, the resulting OMCs possess different sorption effectiveness toward different metal cations. In this report we show that two widely different carbon sources can be used in generating OMC for the sorption of metal cations. The first carbon source is a commercial product distributed by MiSWACO company as an additive to drilling fluids used in the oil industry. It is a black powdery carbon material, called C-seal-F. The second carbon source belongs to the class of bituminous coals; it is naturally occurring in the Russian Federation and mined in millions of tons annually. We will refer to these two carbon sources as $\mathrm{C} 1$ and $\mathrm{C} 2$, respectively. The OMC samples made from the two sources will be labeled as OMC1 and OMC2, respectively.

We developed two different oxidation methods to prepare OMC: first with the use of $\mathrm{KMnO}_{4}$ in sulfuric acid media, and second with the use of the $\mathrm{HNO}_{3} / \mathrm{H}_{2} \mathrm{SO}_{4}$ system. The two oxidation methods used toward the same carbon source yield similar OMC products. With $\mathrm{C} 1$ the $\mathrm{KMnO}_{4} / \mathrm{H}_{2} \mathrm{SO}_{4}$ method gave a slightly more oxidized OMC product compared to the $\mathrm{HNO}_{3} / \mathrm{H}_{2} \mathrm{SO}_{4}$ method. With $\mathrm{C} 2$ both methods gave equally oxidized OMC products. The sorption performance of two OMC samples made from the same carbon source with the use of two different methods was in the range of the experimental error. This is why, in this work, we show the data obtained with the use of only one particulate method toward the one carbon source: the $\mathrm{KMnO}_{4} / \mathrm{H}_{2} \mathrm{SO}_{4}$ method used toward $\mathrm{C}$, and the $\mathrm{HNO}_{3} / \mathrm{H}_{2} \mathrm{SO}_{4}$ method used toward C2.

\section{Experimental Part}


2.1. Materials. Sulfuric acid was from "Shchekinoazot" Trading House, LLC, Russia; nitric acid was from CJSC "TatHimProduct", Russia; potassium permanganate was from MCD Company, Russia. The carbon source C1 was grade C-seal-F from MiSWACO company, USA. The carbon source C2 was a sample of bituminous coal mined in the Karelia region, Russia. Only nonradioactive metal cations were used in this study

2.2. Characterization. The scanning electron microscopy (SEM) images were acquired with a field-emission high-resolution scanning electron microscope Merlin from Carl Zeiss at accelerating voltage of incident electrons of $5 \mathrm{kV}$ and a current probe of $300 \mathrm{pA}$. The X-ray photoelectron spectroscopy (XPS) spectra were acquired in a UHV chamber of the multitechnique surface analysis system Phoibos 100/150 from SPECS. The Mg Ka X-ray source operated at $12.5 \mathrm{kV}$ and $250 \mathrm{~W}$ was used. A pass energy of $30 \mathrm{eV}$ (step size of $0.5 \mathrm{eV}$ ) was used for wide range scans; pass energy of $20 \mathrm{eV}$ (step size of $0.1 \mathrm{eV}$ ) was used for high resolution measurements. All spectra were analyzed by using the CasaXPS software. The powder X-ray diffraction (XRD) was acquired with Bruker D8 Advance with $\mathrm{Cu} \operatorname{K\alpha }$ irradiation $(\lambda=1.5418 \AA$ ) in the Bragg-Brentano geometry; the rate was $0.18 \% \mathrm{~min}$; the range of $2 \theta$ angle was from $7^{\circ}$ through $57^{\circ}$; the step was $0.015^{\circ}$. The thermogravimetric analysis (TGA) data was collected with the Q50 analyzer from TA Instruments in Ar atmosphere with the heating rate of $10 \% \mathrm{~min}$. The content of metal cations was determined with the ionically coupled plasma mass-spectrometer (ICP MS) "iCAP Qc" from Thermo Fisher Scientific.

\subsection{Preparation of OMC}

To prepare OMC1, we first prepared the $88.6 \%$ sulfuric acid by diluting $150 \mathrm{~g} 96 \%$ commercial sulfuric acid with $12.5 \mathrm{~g}$ DI water. The diluted acid was cooled to the room temperature. $10.0 \mathrm{~g}$ of $\mathrm{C} 1$ were introduced into the $88.6 \%$ sulfuric acid with stirring. Potassium permanganate $\left(\mathrm{KMnO}_{4}\right)$ was introduced in two portions of $6.0 \mathrm{~g}$ each with a $1 \mathrm{~h}$ interval. The reaction mixture was continuously stirred for $12 \mathrm{~h}$ after the last $\mathrm{KMnO}_{4}$ portion. Next, the reaction was quenched with $500 \mathrm{~mL}$ DI water, and the diluted reaction mixture was filtered to separate OMC1 from the acidic waste. The OMC1, collected from the filter, was redispersed in $500 \mathrm{~mL}$ DI water, stirred for 30 min with a magnetic stirrer, and the dispersion was filtered to separate the purified OMC1. The above mentioned purification procedure was repeated $4 \mathrm{x}$. The filtrate was always transparent and colorless indicating that very little submicron-scale particles were dispersed in 
water, and that carbon did not crush to smaller fragments even with magnetic stirring. After the $5^{\text {th }}$ washing, the $\mathrm{pH}$ of the filtrate was $\sim 5$ indicating removal of practically all the sulfuric acid. The as-prepared OMC1 was dried in open air until no change in weight was registered. The $13.49 \mathrm{~g}$ of the product was obtained.

To prepare OMC2, $10.0 \mathrm{~g} \mathrm{C} 2$ was introduced into the mixture of $100 \mathrm{~g} 96 \%$ sulfuric acid and 25 $\mathrm{mL} 63 \%$ nitric acid. The reaction was stirred for $12 \mathrm{~h}$. Separation and purification of the asobtained OMC2 were conducted the same way as for the OMC1. The $7.48 \mathrm{~g}$ of the OMC2 product was obtained.

\subsection{Sorption Experiments}

To prepare "contaminated" water, we artificially introduced certain amounts of non-radioactive isotopes of $\mathrm{Cs}^{+}$and $\mathrm{Sr}^{2+}$ into the natural spring water. Also, salts of the $\mathrm{Eu}^{3+}, \mathrm{Gd}^{3+}$ and $\mathrm{Tb}^{3+}$ were added as the model elements for radioactive $\mathrm{U}$ and Am. The nitrates salts were used in all the cases. The original concentration of all the salts in the as-prepared ion-enriched natural spring water was $1 * 10^{-6} \mathrm{M}$. In a typical sorption experiment, a certain amount of a sorbent was introduced into $100 \mathrm{~mL}$ of the artificially ion-enriched water, and stirred for $2 \mathrm{~h}$ to afford sorption of metal ions by the sorbent. Then, the sorbent was separated from water by filtration through a cellulose acetate membrane. The content of as-purified water was compared against the original water. The concentration of metal cations in water samples was determined by ICP MS.

\section{Results and Discussion}



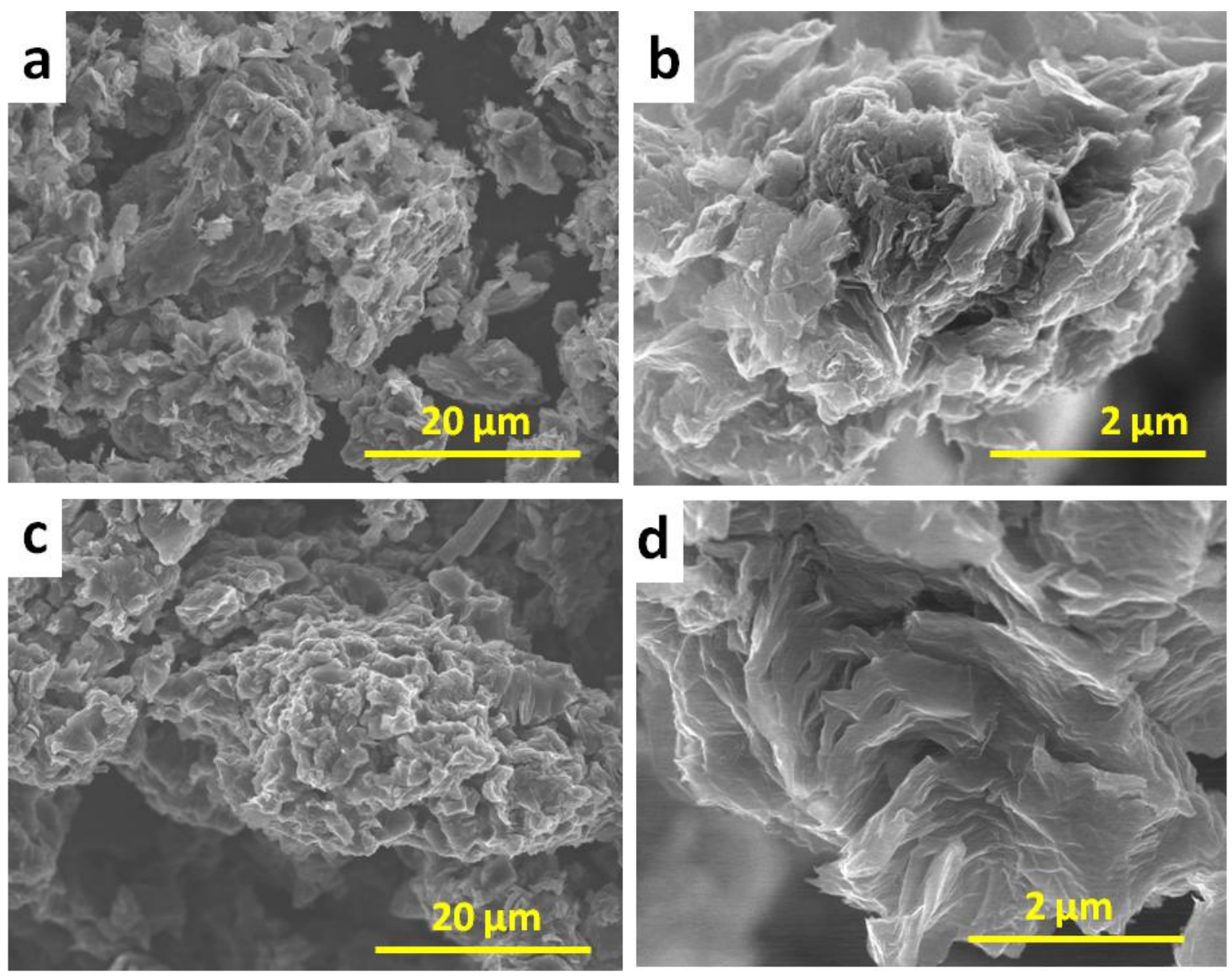

Figure 1. SEM images of $(\mathrm{a}, \mathrm{b}) \mathrm{C} 1$ and $(\mathrm{c}, \mathrm{d}) \mathrm{OMC1}$. The particulate nature of the carbon source is preserved after the oxidation. 

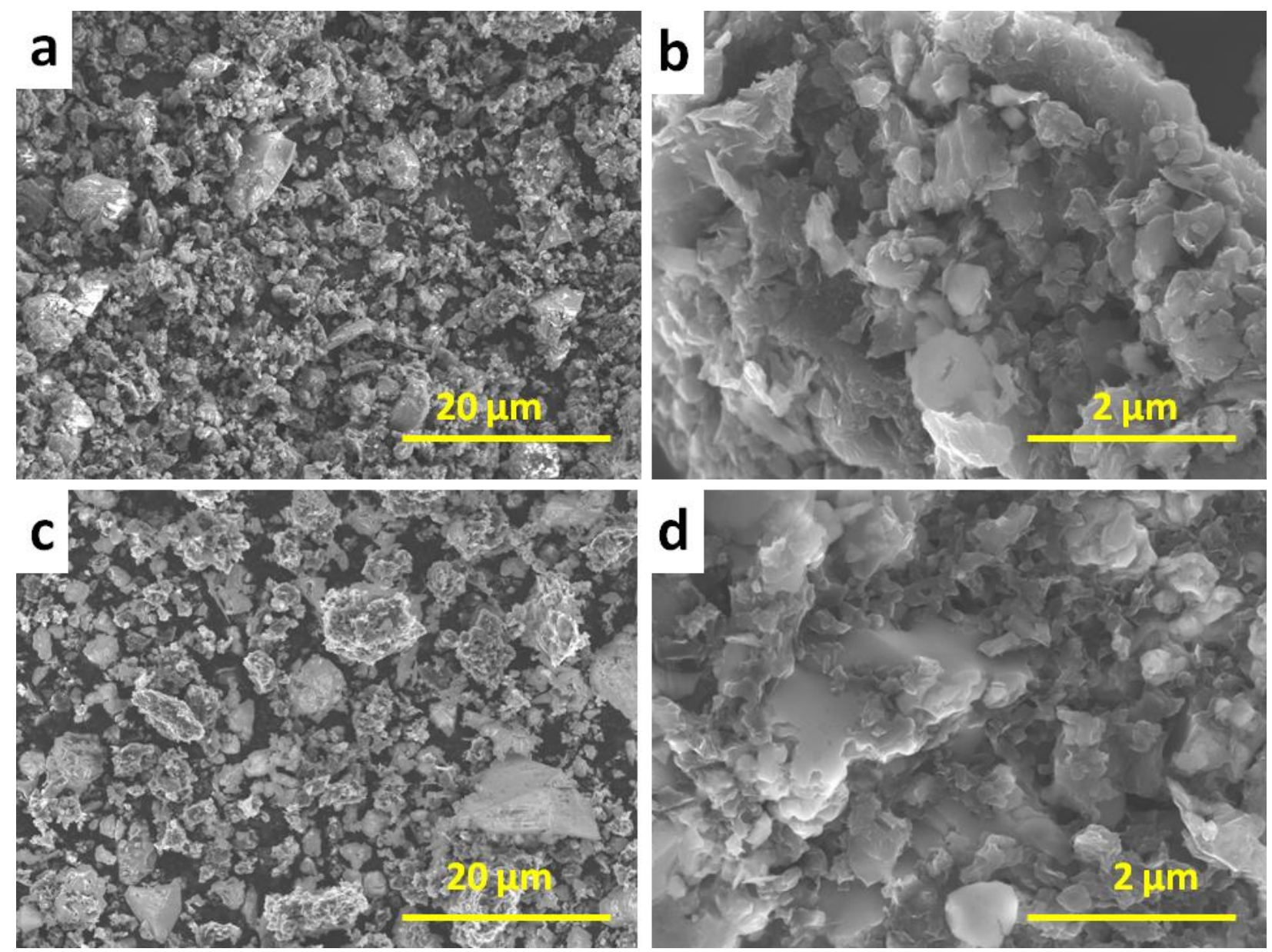

Figure 2. SEM images of (a,b) $\mathrm{C} 2$ and $(\mathrm{c}, \mathrm{d}) \mathrm{OMC}$. The particulate nature of the carbon source is preserved after the oxidation.

The SEM images demonstrate that the $\mathrm{C} 1$ particles appear in the form of crumpled-paper-like balls, or rose flowers with an irregular and highly developed surface (Fig. 1a,b). The particle size varies from $10 \mu \mathrm{m}$ through $80 \mu \mathrm{m}$. Oxidation does not notably change the appearance of the particles (Fig 1c,d); thus OMC1 retains the particulate morphology of the precursor. C2 consists of two types of particles: those appearing similar to the particles of $\mathrm{C} 1$, and particles that appear as uniform solid bodies (Fig. 2 a,b). Similar to C2, oxidation does not change appearance of the sample. OMC2 retains its granular particulate nature (Fig. 2c,d). 


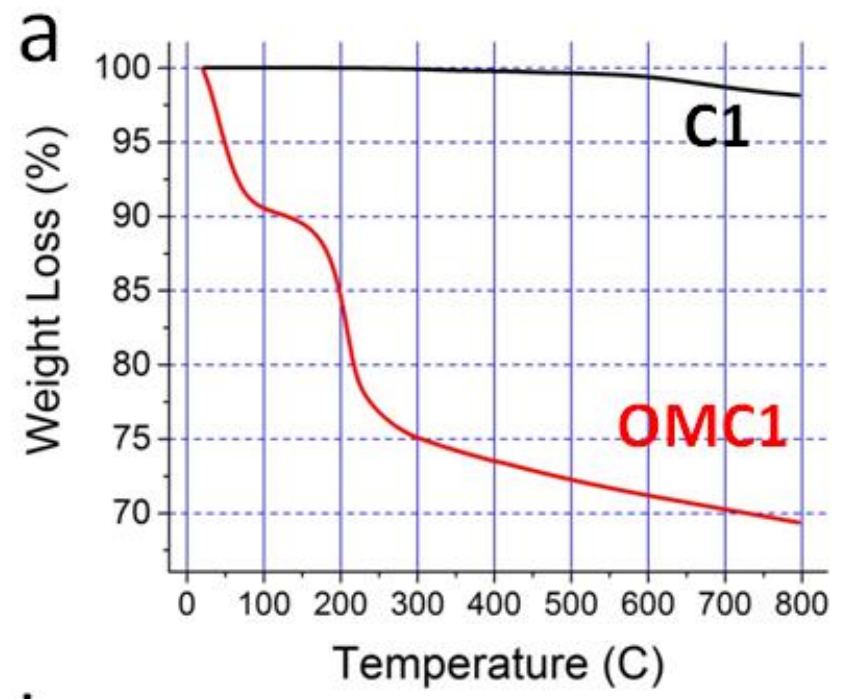

b

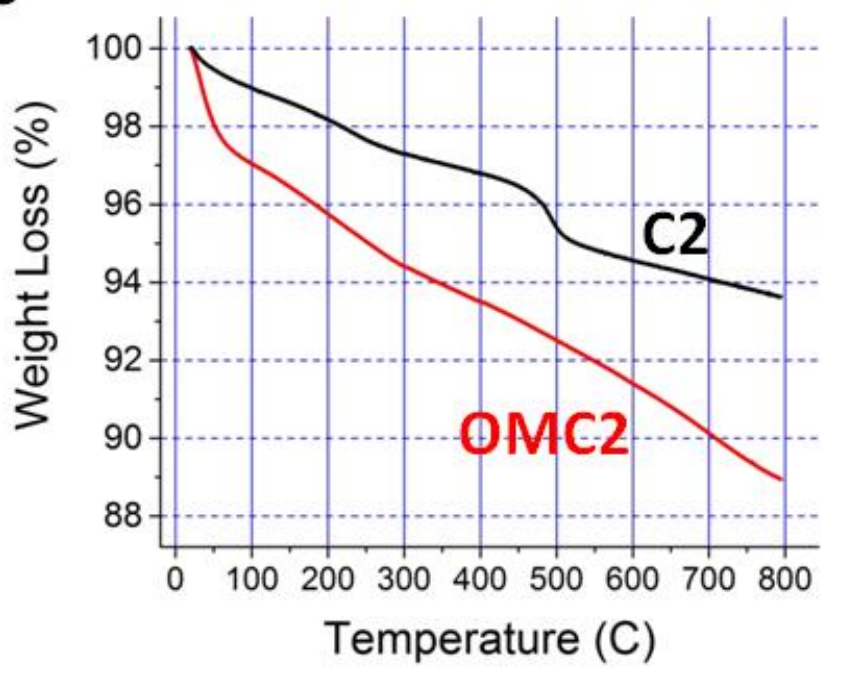

Figure 3. The TGA curves for $\mathrm{C} 1, \mathrm{C} 2, \mathrm{OMC} 1$ and $\mathrm{OMC} 2$. The analysis was carried out under $\mathrm{Ar}$ at the heating rate $10 \mathrm{~K} / \mathrm{min}$.

Figure 3 represents the TGA data for two carbon sources and the two OMC samples made from these sources. $\mathrm{C} 1$ loses almost no weight until $600^{\circ} \mathrm{C}$, and only $2 \%$ weight loss is registered between $600^{\circ} \mathrm{C}$ and $800^{\circ} \mathrm{C} \mathrm{(Fig.} \mathrm{3a).} \mathrm{This} \mathrm{is} \mathrm{typical} \mathrm{for} \mathrm{carbon} \mathrm{sources} \mathrm{with} \mathrm{high} \mathrm{degree} \mathrm{of}$ graphitization. $\mathrm{OMC} 1$ exhibits $8 \%$ weight loss by $100^{\circ} \mathrm{C}$ associated with the loss of absorbed water, and the definitive $13 \%$ weight loss in the $160^{\circ} \mathrm{C}$ to $220^{\circ} \mathrm{C}$ temperature interval, associated with decomposition of the oxygen functional groups. Such behavior, typical for $\mathrm{GO},{ }^{13}$ is indicative of the high oxidation level of OMC1. 
$\mathrm{C} 2$ exhibits monotonous $6 \%$ weight loss in the entire temperature interval, with the sharp $1.5 \%$ weight loss at $\sim 490^{\circ} \mathrm{C}$ (Fig. 3b). The latter is most likely associated with evaporation of residual non-graphitized bitumen. The OMC2 exhibits $2.5 \%$ weight loss by $100^{\circ} \mathrm{C}$, and monotonous $8.5 \%$ weight loss between $100^{\circ} \mathrm{C}$ and $800^{\circ} \mathrm{C}$. The $\sim 2.5 \%$ weight loss (loss of the absorbed water) by OMC2 points at the oxidized and thus hydrophilic nature of its surface. However, there is only insignificant weight loss in the $160^{\circ} \mathrm{C}$ to $220^{\circ} \mathrm{C}$ temperature interval, suggesting that the overall oxidation level of $\mathrm{OMC} 2$ is much lower than that of $\mathrm{OMC} 1$.

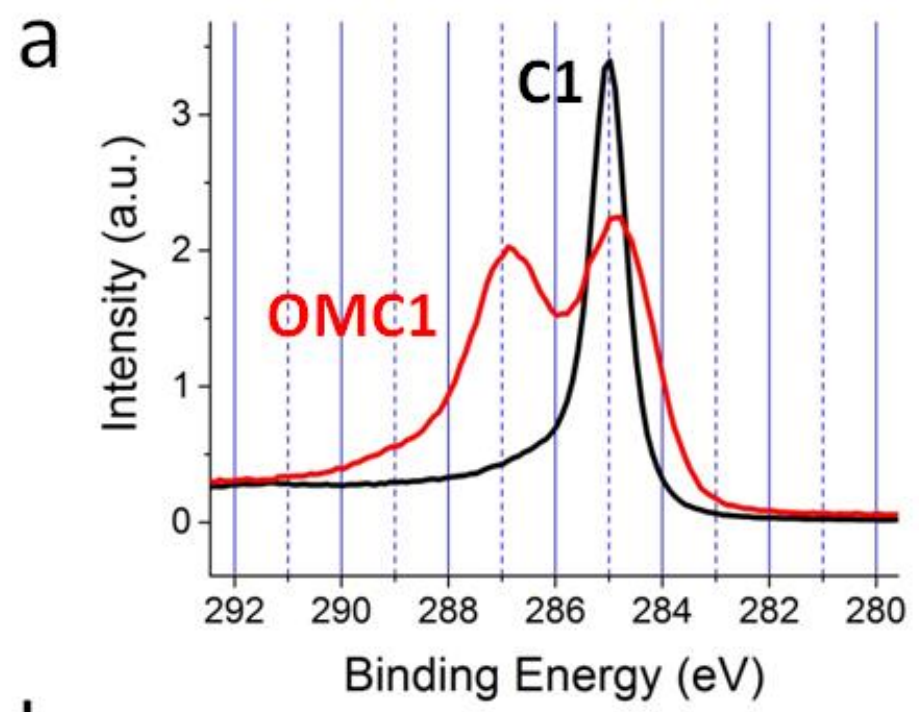

b

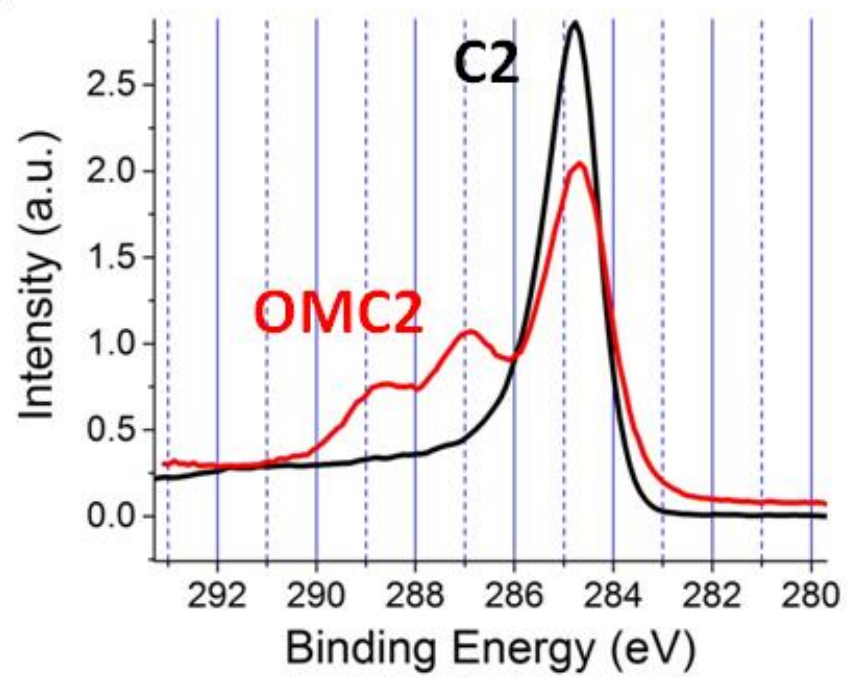

Figure 4. The C1s XPS spectra for $\mathrm{C} 1, \mathrm{C} 2, \mathrm{OMC} 1$ and $\mathrm{OMC} 2$. 
Similar conclusion can be made from the XPS data for the two samples (Fig 4). The C1s spectrum of OMC1 (Fig. 4a) contains two components: the one centered at $284.8 \mathrm{eV}$ is associated with non-oxidized carbon atoms, the one centered at $286.9 \mathrm{eV}$ is attributed to carbon atoms of alcohol $\mathrm{C}-\mathrm{OH}$ and epoxide $\mathrm{C}-\mathrm{O}-\mathrm{C}$ groups. The intensities of the two components are almost equal, suggesting moderately high oxidation levels of the surface. Such an oxidation level is typical for low-to-moderately oxidized GO samples. ${ }^{18} \mathrm{OMC} 2$ has a significantly lower oxidation level (Fig. 4b). However, the sample contains a higher percentage of carboxyls, which can play a key role in sorption of metal cations. Thus, the intensity of the $289 \mathrm{eV}$ component (carboxyls and carbonyls) is comparable to that of the $286.9 \mathrm{eV}$ component (alcohols and epoxides).

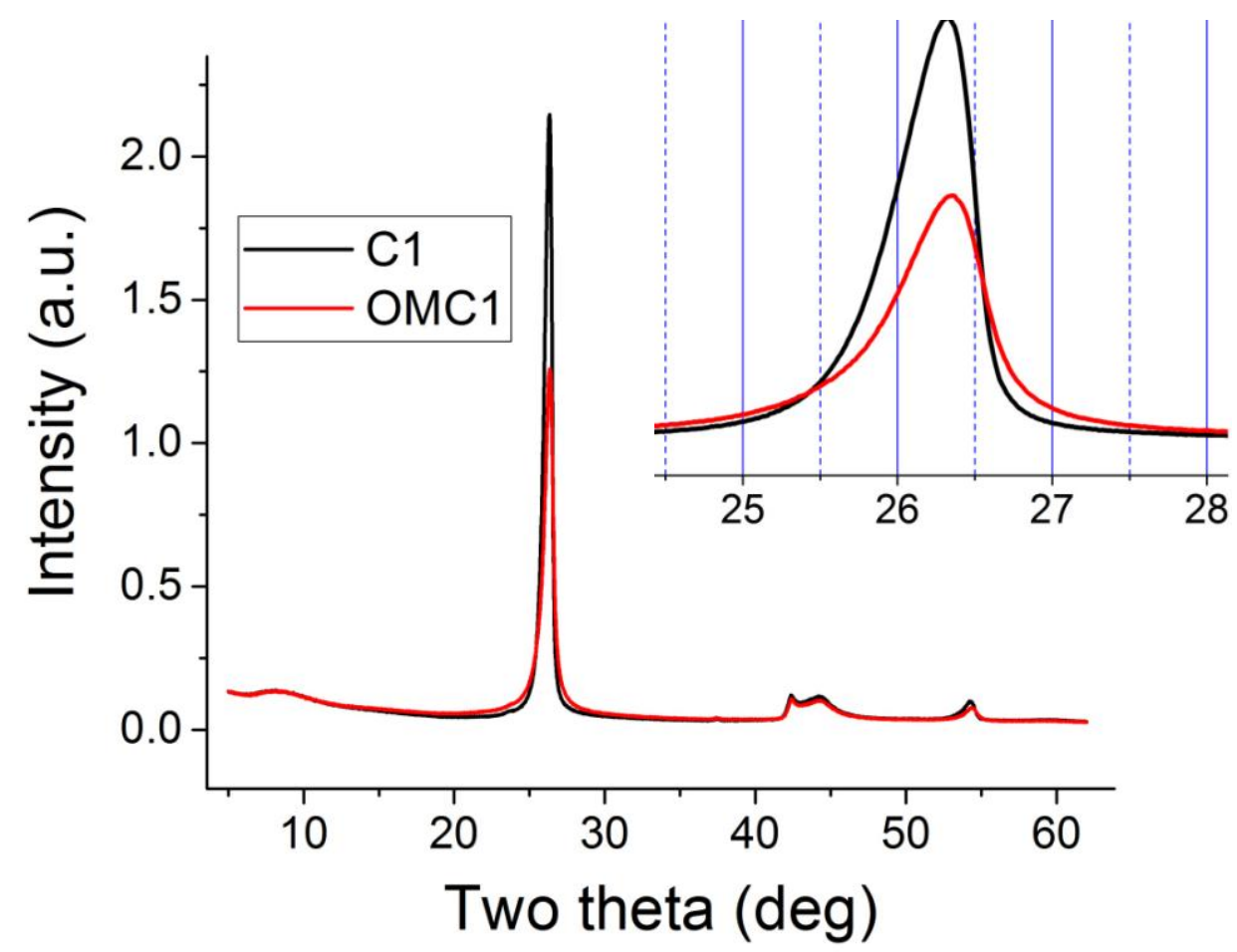

Figure 5. The XRD pattern for $\mathrm{C} 1$ and $\mathrm{OMC} 1$. The inset is the $\mathrm{X}$-axis expansion of the 002 signal area.

The X-ray powder diffraction (XRD) pattern of C1 shows characteristic graphitic signal at $26.3^{\circ}$ $2 \theta$ angle (Fig. 5), suggesting the highly graphitized nature of the material. After oxidation, this signal lowers in intensity and broadens, manifesting some decrease in crystallinity. Also the 002 signal of OMC1 has two well-pronounced wings on the left and on the right. This might be due 
to superposition of two different signals: a high and sharp peak from graphite, and a low and broad peak from another form of carbon. Liu et al. demonstrated that such a shape of the 002 peak is typical for the ordered mesoporous carbon. ${ }^{19}$ Thus, most likely OMC1 is a twocomponent system: graphitic core and oxidized mesoporous shell. The indirect evidence for such an assumption comes from the measured BET surface area. While C1 has a BET surface area of $12.4 \mathrm{~m}^{2} / \mathrm{g}$, the OMC1 exhibits $14.1 \mathrm{~m}^{2} / \mathrm{g}$; this data suggests that some additional pores develop during the oxidation.

The XRD pattern for C2 (Fig. S1) shows two different phases. The 002 signal at $26.6^{\circ} 2 \theta$ suggests the presence of the graphitic form. Interestingly, the signal is significantly narrower compared to that of $\mathrm{C} 1$. We were unable to identify another phase. After oxidation the 002 signal does not notably change. Interestingly, the signals, associated with another phase, do not fade away after oxidation, but transfer practically unchanged into the diffraction pattern of OMC2. This suggests that another phase is not a metal oxide (it would dissolve in acidic media during the oxidation), but another form of carbon. When burning out all the carbon (TGA run in air), C2 retains only $1.5 \%$ of its original weight, while $\mathrm{C} 1$ burns down to $\sim \%$. This data demonstrates that $\mathrm{C} 2$ contains an insignificant amount of metal ores, while $\mathrm{C} 1$ contains none.

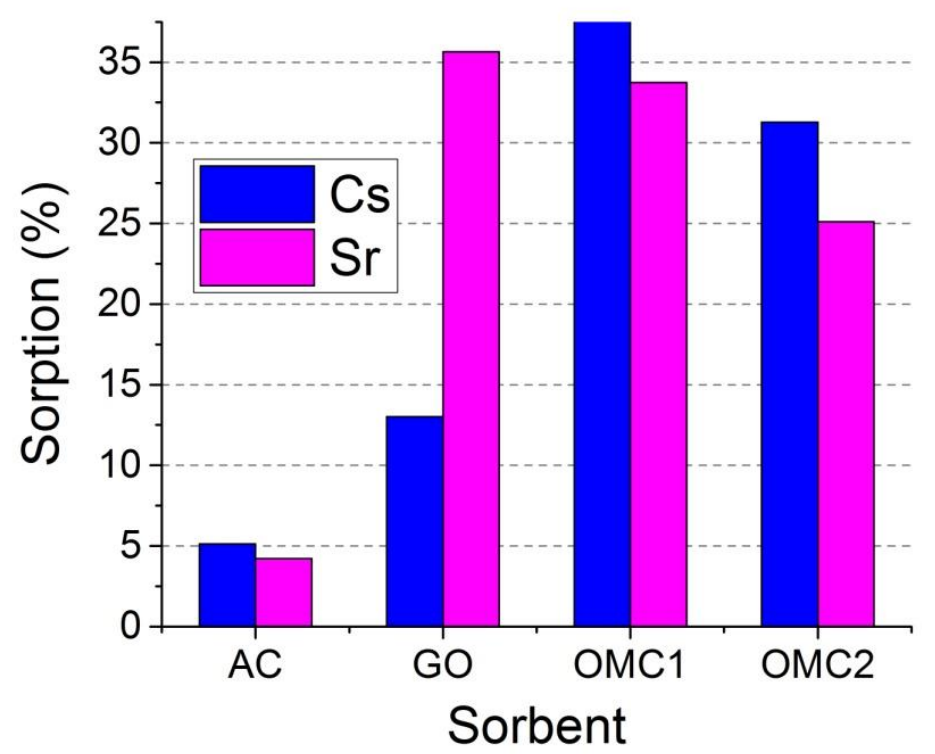

Figure 6. Sorption abilities of different carbon-based materials. The two OMC samples are compared to activated carbon (AC) and graphene oxide (GO). $50 \mathrm{mg}$ of sorbent was used to 
purify $100 \mathrm{~mL}$ of the $\mathrm{Cs}^{+}$and $\mathrm{Sr}^{2+}$ enriched natural spring water. The original concentration of the $\mathrm{Cs}^{+}$and $\mathrm{Sr}^{2+}$ cations were each $1 * 10^{-6} \mathrm{M}$.

We intended to demonstrate the efficiency of the as-prepared OMC samples in a realistic system, i.e. toward water from natural reservoirs. This is why we have chosen natural spring water as the "baseline" water source. To prepare "contaminated" water we artificially introduced certain amounts of non-radioactive isotopes of $\mathrm{Cs}^{+}$and $\mathrm{Sr}^{2+}$ into the natural spring water. Also, salts of the $\mathrm{Eu}^{3+}, \mathrm{Gd}^{3+}$ and $\mathrm{Tb}^{3+}$ were added as the model elements for radioactive $\mathrm{U}$ and $\mathrm{Am}$. The nitrates salts were used in all the cases. The original concentration of all the salts was $1 * 10^{-6} \mathrm{M}$, i.e. close to that in the waters of the Fukushima nuclear plant. ${ }^{1}$ Note, at such low concentrations, the five artificially added elements competed with cations naturally present in the spring water. The content of some of them such as $\mathrm{Na}^{+}, \mathrm{K}^{+}, \mathrm{Ca}^{2+}, \mathrm{Mg}^{2+}$ exceeded the content of the five target elements by one to two orders in magnitude. We monitored the content of almost all the metals present in water samples. Even though we studied five different elements, our main target was $\mathrm{Cs}^{+}$.

We demonstrate the sorption ability of the two prepared OMC samples in comparison to that of the known carbon-based sorbents (Fig. 6). In this experiment we introduced $50 \mathrm{mg}$ of the sorbent into $100 \mathrm{~mL}$ of the artificially ion enriched water, stirred for $2 \mathrm{~h}$ and then separated the sorbent from water by filtration. The content of as-purified water was compared against the original water. Activated carbon (AC) exhibits very low sorption capacity toward metal cations (Fig. 6). This is expected: while AC is efficient toward gaseous substances and organic compounds due to its highly developed surface area, carbon in general is passive toward charged metal cations.

Oxygen functional groups, as in GO, are needed to render material active toward metal cations. GO is efficient toward Sr, but not as much toward Cs. Surprisingly, both OMC samples exhibit very high selectivity toward Cs, outperforming even GO. The selectivity of the samples toward $\mathrm{Sr}$ is lower than that toward Cs. This is puzzling since binding of divalent cations to the oxidized carbon surface should be stronger compared to monovalent cations.

To demonstrate the full potential of the two OMC samples toward different metal cations, we investigated efficiencies of these sorbents at different loadings. The sorbent samples of $50 \mathrm{mg}$, $100 \mathrm{mg}, 200 \mathrm{mg}, 400 \mathrm{mg}$ and $800 \mathrm{mg}$ were applied toward $100 \mathrm{~mL}$ water. The rest of the 
procedure was the same as in the previous experiment. Sorption efficiency of the two carbon precursors $\mathrm{C} 1$ and $\mathrm{C} 2$ was investigated along with the two OMC samples.

Figure 7 demonstrates that removal of metal cations is not linear with loading, as one might expect, but exponential. This is because the amount of sorbent is indirectly present in the Langmuir isotherm equation.
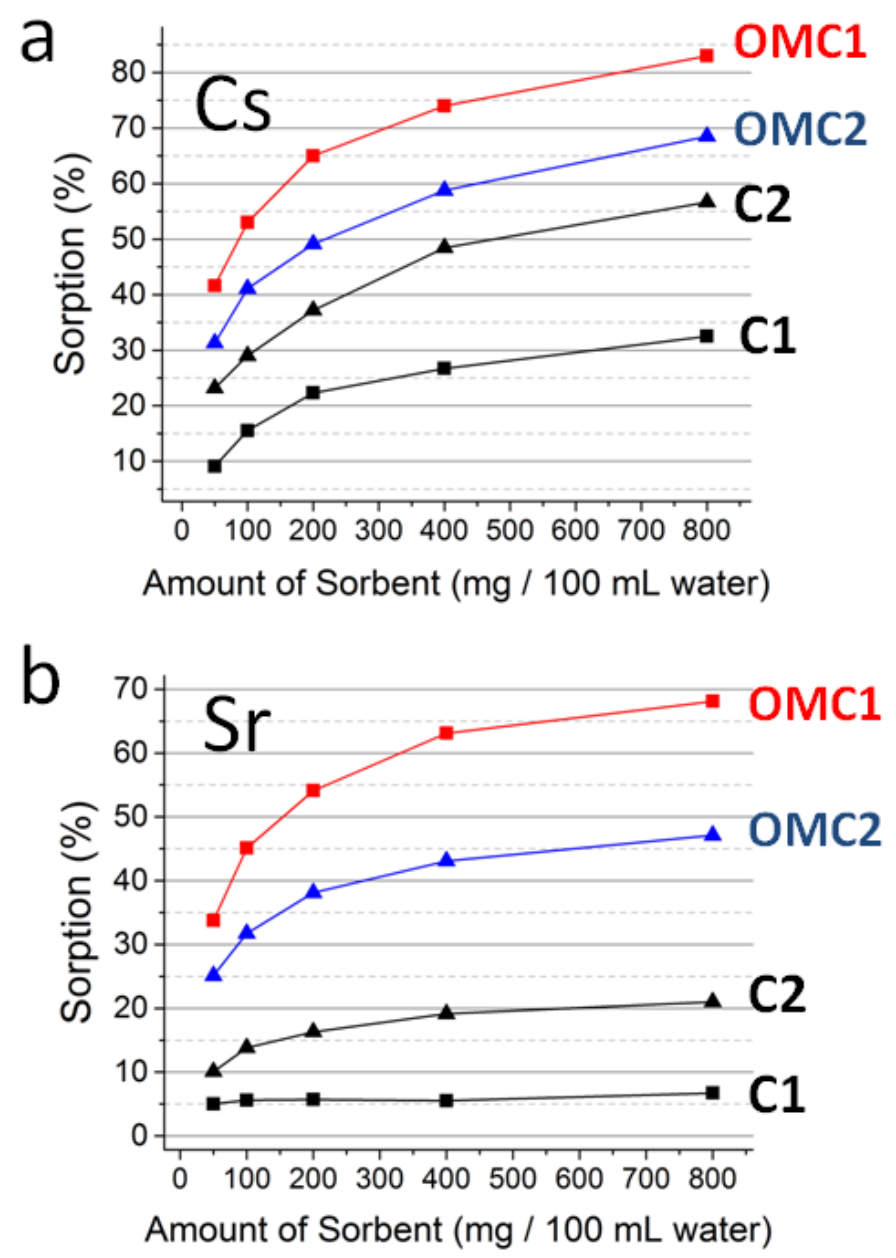

Figure 7. Sorption of the $\mathrm{Cs}^{+}$and $\mathrm{Sr}^{2+}$ cations by four different sorbents. Initial concentration of the $\mathrm{Cs}^{+}$and $\mathrm{Sr}^{2+}$ ions were $1 * 10^{-6} \mathrm{M}$ each. The $\mathrm{Y}$-axis represents percentage of metal cations removed from water out of their original content.

Surprisingly, the affinity of the both nonoxidized carbon precursors toward cesium is not zero (Fig. 7a). This is especially true for $\mathrm{C} 2$ that can absorb almost as much $\mathrm{Cs}^{+}$as its oxidized counterpart: the $\mathrm{Cs}^{+}$sorption by $\mathrm{OMC} 2$ is only slightly better than that by $\mathrm{C} 2$. The difference in 
sorption ability between $\mathrm{C} 2$ and OMC2 is not that large as between $\mathrm{C} 1$ and OMC1. This suggests that at least part of the OMC2 affinity toward $\mathrm{Cs}^{+}$is due to the structure of the carbon precursor itself, but is not solely due to the surface oxygen groups.

Surprisingly, sorption ability toward divalent $\mathrm{Sr}^{2+}$ (Fig. 7b) is lower compared to that for monovalent $\mathrm{Cs}^{+}$. This is unexpected for an oxidized carbon material, considering that GO sorbs $\mathrm{Sr}^{2+}$ more effectively than $\mathrm{Cs}^{+}$(Fig. 6).

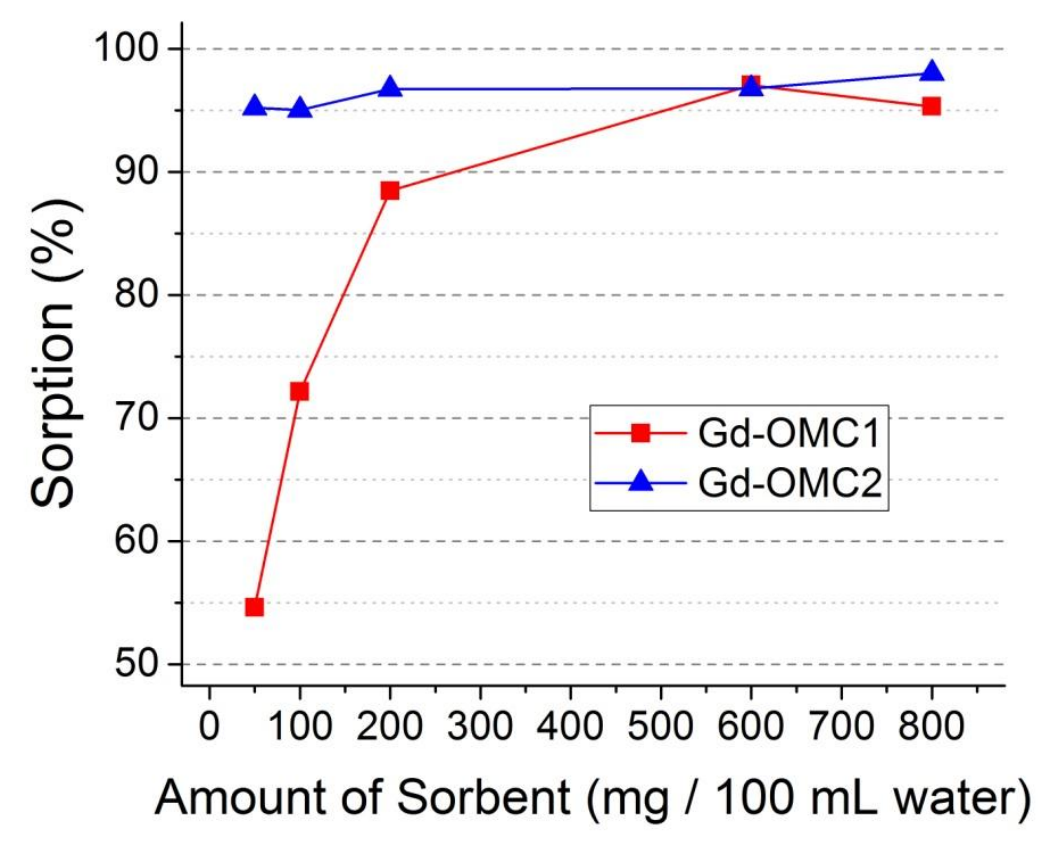

Figure 8. Sorption efficiencies of OMC1 and OMC2 toward Gd. The Y-axis represents percentage of metal cations removed from solution out of the original metal content.

Next, we explored efficacy of OMC toward the three lanthanides $\mathrm{Eu}^{3+}, \mathrm{Gd}^{3+}$ and $\mathrm{Tb}^{3+}$. Sorption of all the three was very similar. Figure 8 shows sorption of $\mathrm{Gd}^{3+}$, and Figures S2 and S3 show sorption of $\mathrm{Eu}^{3+}$ and $\mathrm{Tb}^{3+}$ respectively. Expectedly, removal of these three elements is higher than that for $\mathrm{Cs}^{+}$and $\mathrm{Sr}^{2+}$. Surprisingly, OMC2 was more efficient than OMC1 toward all the three elements. 


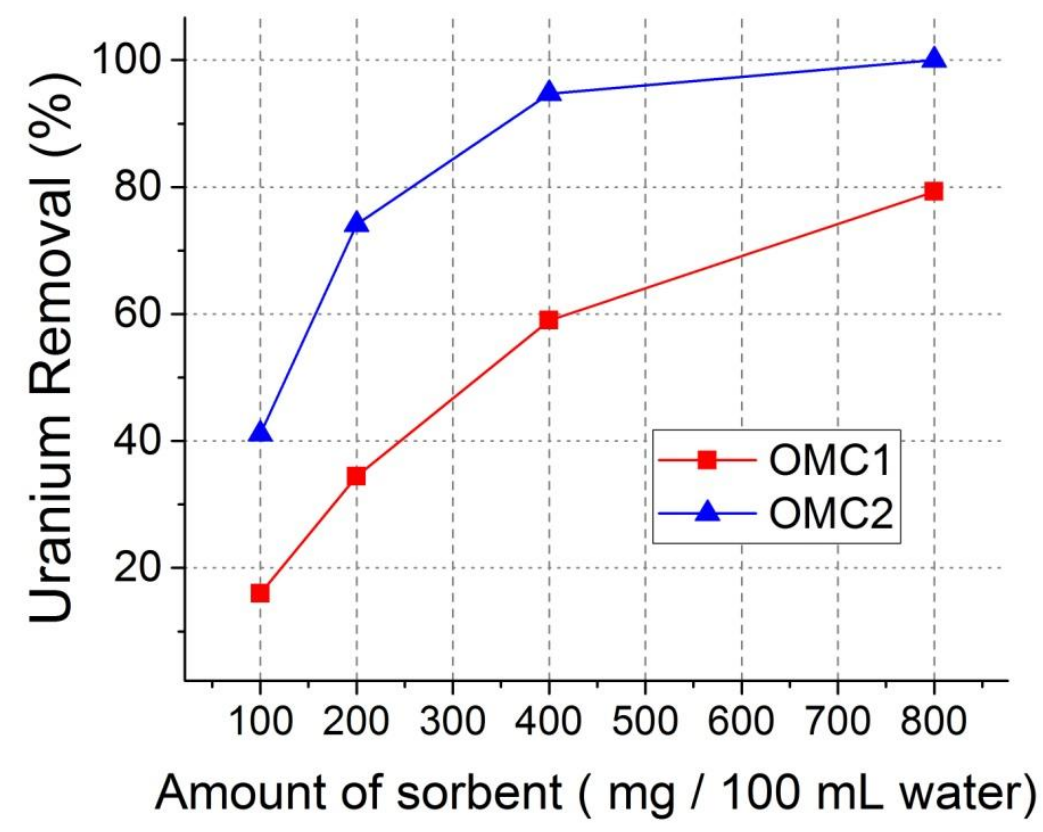

Figure 9. Sorption efficiencies of $\mathrm{OMC} 1$ and $\mathrm{OMC} 2$ toward $\mathrm{U}$. The $\mathrm{Y}$-axis represents percentage of metal cations removed from solution out of the original metal content

Finally, we tested the sorption efficacy of the two OMC samples toward uranium that is naturally present in water. The original concentration of uranium was only $0.222 \mathrm{ppb}$, which is approximately 1000x lower than the content of the five $\left(\mathrm{Cs}^{+}, \mathrm{Sr}^{2+}, \mathrm{Eu}^{3+}, \mathrm{Gd}^{3+}\right.$ and $\left.\mathrm{Tb}^{3+}\right)$ artificially added ions, four orders of magnitude lower than $\mathrm{K}^{+}$and $\mathrm{Na}^{+}$, and five orders of magnitude lower than $\mathrm{Ca}^{2+}$, naturally present in the spring water. The uranium sorption was higher than that for $\mathrm{Cs}^{+}$and $\mathrm{Sr}^{2+}$, but lower than that for $\mathrm{Eu}^{3+}, \mathrm{Gd}^{3+}$ and $\mathrm{Tb}^{3+}$. Again, OMC2 was more efficient toward uranium than OMC1. 


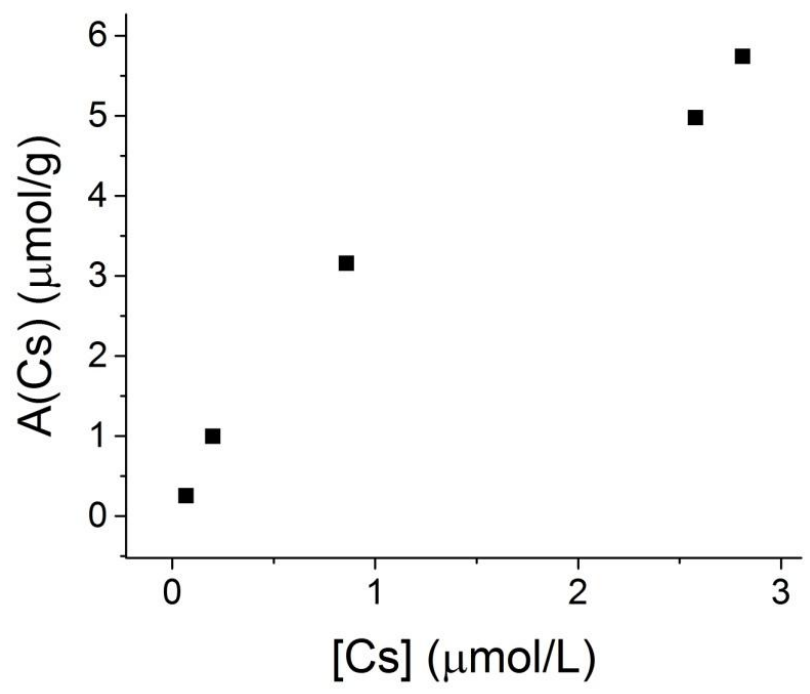

Figure 10. Sorption isotherm for $\mathrm{Cs}^{+}$ions. The $\mathrm{Y}$-axis is the number of moles $\mathrm{Cs}^{+}$absorbed by $1.0 \mathrm{~g}$ of sorbent $\mathrm{A}(\mathrm{Cs})$. The $\mathrm{X}$-axis is the equilibrium concentration of $\mathrm{Cs}^{+}$in water.

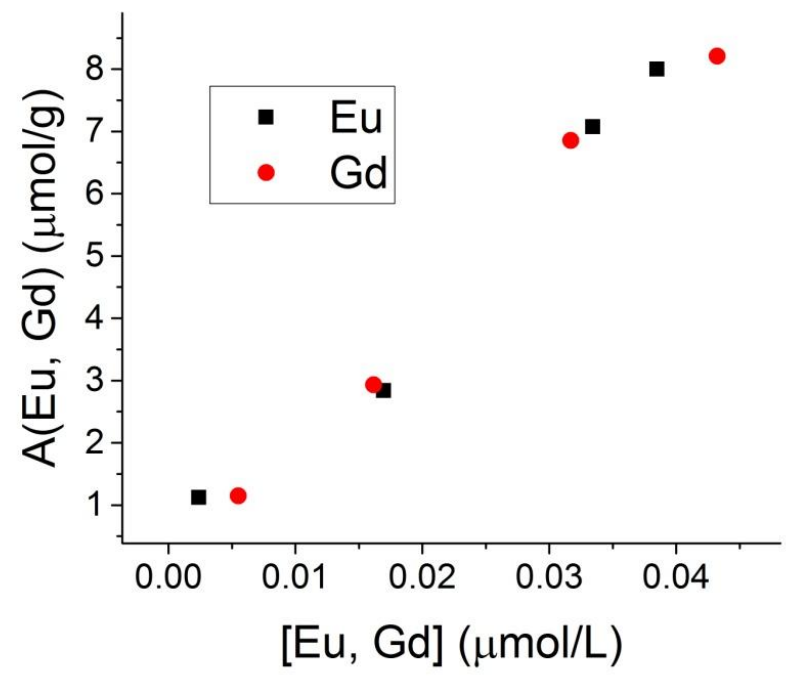

Figure 11. Sorption isotherm for $\mathrm{Eu}^{3+}$ and $\mathrm{Gd}^{3+}$ ions. The $\mathrm{Y}$-axis is the number of moles $\mathrm{Eu}^{3+}$ and $\mathrm{Gd}^{3+}$ absorbed by $1.0 \mathrm{~g}$ of sorbent $\mathrm{A}(\mathrm{Eu}, \mathrm{Gd})$. The $\mathrm{X}$-axis is the equilibrium concentration of metal ions in water. 
Figures 10, 11 and Figure S4 represent sorption isotherms as the function of the moles of metals adsorbed by $1.0 \mathrm{~g}$ of sorbent from the equilibrium (remaining) concentration of metals in solution. To obtain these isotherms, we conducted additional experiments where the original concentration of metal cations varied at constant amount of the sorbent. The isotherms for $\mathrm{Eu}^{3+}$, $\mathrm{Gd}^{3+}$ and $\mathrm{Tb}^{3+}$ are almost identical. The isotherms for these three elements and that for $\mathrm{Sr}^{2+}$ can be classified as the S-type according to Giles. ${ }^{20}$ The isotherm for $\mathrm{Cs}^{+}$can be classified as the Ltype. The mathematical description of the isotherms is impossible due to the multi-component character of the system with numerous competing metal ions.

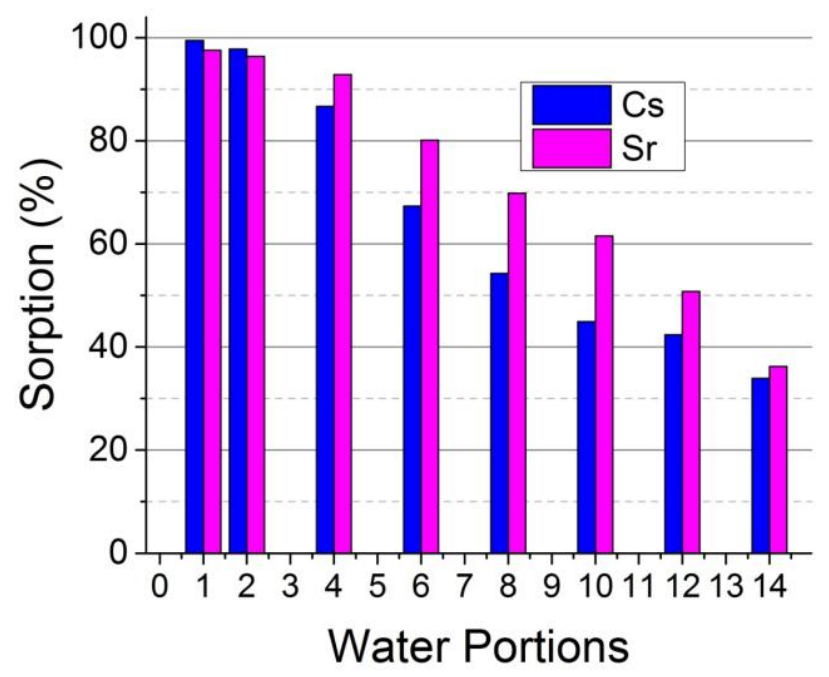

Figure 12. Removal of $\mathrm{Cs}^{+}$and $\mathrm{Sr}^{2+}$ from ion-enriched spring water by $\mathrm{OMC} 1$ in the column filtration geometry. $1.0 \mathrm{~g}$ of $\mathrm{OMC} 1$ was used in the column as the sorption material. $1400 \mathrm{~mL}$ water was passed through the column in portions of $100 \mathrm{~mL}$ each. The content of every even water portion was determined after the second portion.

As a proof of concept in a flow system, we used OMC1 in a traditional filtration column. Water was passed through a column in portions, and the content of each portion was analyzed separately. $1.0 \mathrm{~g}$ OMC1 was used in the column as the sorption material. The volume of each water portion was $100 \mathrm{~mL}$. The concentration of $\mathrm{Cs}^{+}$and $\mathrm{Sr}^{2+}$ in each portion of water was $1^{*} 10^{-}$ ${ }^{6} \mathrm{M}$. The water flow through the column was under gravity force. It took $\sim 10 \mathrm{~min}$ for $100 \mathrm{~mL}$ of water to pass through the column. As evident from Fig. 12, removal of metals is very efficient with the first several water portions. However, the sorption notably decreases with every new 
water portion. Removal of trivalent $\mathrm{Eu}^{3+}, \mathrm{Gd}^{3+}$ and $\mathrm{Tb}^{3+}$ was above $90 \%$ even in the $14^{\text {th }}$ tested water portion.

Next, we examined desorption of the target metal cations. After the $14^{\text {th }}$ water portion the column was washed with $7 \mathrm{~mL}$ of $0.05 \mathrm{M} \mathrm{HCl}$. This procedure caused the removal of $92.6 \%$ and 91.3\% of adsorbed $\mathrm{Cs}^{+}$and $\mathrm{Sr}^{2+}$, respectively. Thus, we concentrated the $\mathrm{Cs}^{+}$and $\mathrm{Sr}^{2+}$ contaminated water by 200: from $1400 \mathrm{~mL}$ down to $7 \mathrm{~mL}$. In principle, this dilute acid can be neutralized and evaporated leaving a concentrated radioactive sediment. This experiment demonstrates that $\mathrm{OMC}$ can be used as an ion-exchange resin in traditional sorption columns.

\section{Acknowledgments:}

The work is performed with support of the Russian Government Program of Competitive Growth of Kazan Federal University. SEM images were acquired using the equipment of Interdisciplinary Center for Analytical Microscopy, Kazan (Volga Region) Federal University.

\section{References:}

1. Kumamoto Y, Aoyama M, Hamajima Y, Murata A, Kawano T Impact of Fukushimaderived radiocesium in the western North Pacific Ocean about ten months after the Fukushima Daiichi nuclear power plant accident. J.Environ.Radioact. 2015, 140, 114 122.

2. Choppin, G,R,: Khankhasayev, M.K. Chemical separation technologies and related methods of nuclear waste management: applications problems and research needs. Kluwer, Dordrecht, 1999

3. Dubourg, M. Review of advanced methods for treating radioactive contaminated water. Radioprotection 1998, 33, 35-46.

4. Abdel Rahman, R.O.; Ibrahium, H.A.; Hung, Y.T. Liquid radioactive wastes treatment: a review. Water 2011, 3, 551-565.

5. Cornel, R.M. Adsorption of cesium on minerals: a review. J. Radioanal. Nucl. Chem. 1993, 171, 483-500.

6. Shakir, K., Sohsah, M., Soliman, M. Removal of cesium from aqueous solutions and radioactive waste simulants by coprecipitate flotation. Sep. Purif. Technol. 2007, 54, 373-381. 
7. Noli, F.; Buema, G.; Misaelides, P. Retention of cesium from aqueous solutions using synthetic zeolites produced from power plant ash. J. Radioanal. Nucl. Chem. 2016, 309, 589-596.

8. Lee, K-Y.; Park, M.; Kim, J.; Oh, M; Lee, E.H.; Kim, K.W.; Chung, D.Y.; Moon, J.K. Equilibrium, kinetic and thermodynamic study of cesium adsorption onto nanocrystalline mordenite from high-salt solution. Chemospere, 2016, 150, 765-771.

9. Vipin, A.K.; Ling, S.; Fugetsu, B. Removal of Cs+ and Sr2+ from water using MWCNT reinforced zeolite-A beads. Microporous Mesoporous Mater. 2016, 224, 84-88.

10. Yildiz, B.; Erten, H.N.; Kis, M. The sorption behavior of Cs ion on clay minerals and zeolite in radioactive waste management: sorption kinetics and thermodynamics. $J$. Radioanal. Nucl. Chem. 2011, 288, 475-483.

11. Kapnisti, M.; Hatzidimitriou, A.; Noli, F.; Pavlidou, E. Investigation of cesium uptake from aqueous solutions using new titanium phosphates ion-exchangers. J. Radioanal. Nucl. Chem. 2015, 303, 2303-2311.

12. Romanchuk, A.Y.; Slesarev, A.S.; Kalmykov, S.N.; Kosynkin, D.V.; Tour, J.M. Graphene oxide for effective radionuclide removal. Phys. Chem. Chem. Phys. 2013, 15, 2321-2327.

13. Dimiev, A.; Alemany, L.; Tour, J.M. Graphene Oxide. Origin of Acidity and Dynamic Structural Model. ACS Nano, 2013, 7, 576-588.

14. Hummers, W. S.; Offeman, R. E. Preparation of graphitic oxide. J. Am. Chem. Soc. 1958, $80(6), 1339$

15. Staudenmaier, L. Verfahren zur darstellung der graphitsäure. Ber. Dtsch. Chem. Ges. 1898, 31 (2), 1481-1487.

16. Kovtyukhova, N. I.; Ollivier, P. J.; Martin, B. R.; Mallouk, T. E.; Chizhik, S. A.; Buzaneva, E. V.; Gorchinskiy, A. D., Layer-by-Layer Assembly of Ultrathin Composite Films from Micron-Sized Graphite Oxide Sheets and Polycations. Chem. Mater. 1999, $11,771-778$.

17. Marcano, D.C.; Kosynkin, D.V.; Berlin, J.M.; Sinitskii, A.; Sun, Z.; Slesarev, A.; Alemany, L.B.; Lu, W.; Tour, J.M. Improved synthesis of graphene oxide. ACS Nano, 2010, 4, 4806-4814. 
18. Hontoria-Lucas, C.; Lopes-Peinado, A.J.; Lopez-Gonzalez, J.D.; Rojas-Cervantes, M.L.; Martin-Aranda, R.M. Study of oxygen-containing groups in a series of graphite oxides: physical and chemical characterization. Carbon, 1995, 33, 1585-1592.

19. Liu, L. et al. Ordered mesoporous carbon catalyst for dehydrogenation of propane to propylene. Chem. Comm. 2011, 47, 8334-8336.

20. Jiles, C.H.; Smith, D. A general treatment and classification of the solute adsorption isotherm, J.Colloid Interf.Sci. 1974, 47, 755-765. 